Document downloaded from:

http://hdl.handle.net/10251/68390

This paper must be cited as:

Martínez-Plumed, F.; Ferri Ramírez, C.; Hernández Orallo, J.; Ramírez Quintana, MJ. (2014). A knowledge growth and consolidation framework for lifelong machine learning systems. 13th International Conference on Machine Learning and Applications (ICMLA 2014). IEEE. doi:10.1109/ICMLA.2014.23.

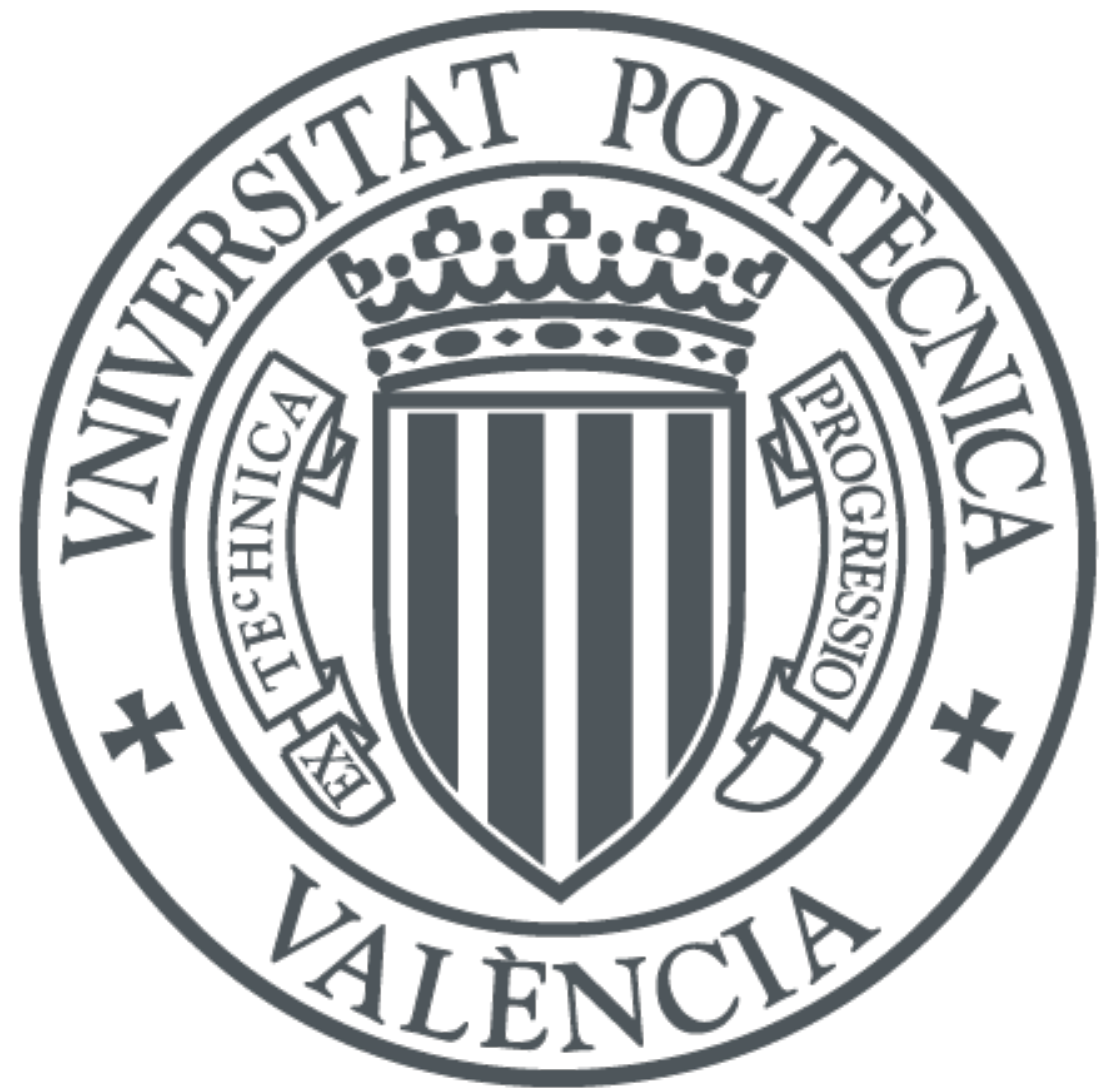

The final publication is available at

http://dx.doi.org/10.1109/ICMLA.2014.23

Copyright IEEE

Additional Information

2014 IEEE. Personal use of this material is permitted. Permission from IEEE must be obtained for all other uses, in any current or future media, including reprinting/republishing this material for advertising or promotional purposes, creating new collective works, for resale or redistribution to servers or lists, or reuse of any copyrighted component of this work in other works. 


\title{
A knowledge growth and consolidation framework for lifelong machine learning systems
}

\author{
Fernando Martínez-Plumed, Cèsar Ferri, José Hernández-Orallo, María José Ramírez-Quintana \\ DSIC, Universitat Politècnica de València, Camí de Vera s/n, 46022 València, Spain. \\ E-mails: $\{$ fmartinez, cferri, jorallo, mramirez\}edsic.upv.es
}

\begin{abstract}
A more effective vision of machine learning systems entails tools that are able to improve task after task and to reuse the patterns and knowledge that are acquired previously for future tasks. This incremental, long-life view of machine learning goes beyond most of state-of-the-art machine learning techniques that learn throw-away models. In this paper we present a long-life knowledge acquisition, evaluation and consolidation framework that is designed to work with any rule-based machine learning or inductive inference engine and integrate it into a long-life learner. In order to do that we work over the graph of working memory rules and introduce several topological metrics over it from which we derive an oblivion criterion to drop useless rules from working memory and a consolidation process to promote the rules to the knowledge base. We evaluate the framework on a series of tasks in a chess rule learning domain.
\end{abstract}

Index Terms-Lifelong machine learning, oblivion criterion, knowledge topology and acquisition, declarative learning.

\section{INTRODUCTION}

The traditional view of machine learning (ML) applications usually portrays a training dataset from which a model is learnt and evaluated and a deployment dataset where the model is applied. Whenever a new application task appears, the old model is discarded and a new one is learnt from a new training set. Recently, different areas, such as transfer learning, multitask learning, lifelong ML, never-ending learners or even deep learning have tried to convert ML systems in more incremental tools able to learn knowledge and partially reuse it for other tasks.

The ingredients of a learning system that is able to acquire knowledge incrementally and arrange it properly in order to improve its performance are the following: (1) an inductive inference engine (generating patterns), (2) a deductive coverage engine (calculating knowledge interdependence), (3) a knowledge quality analyser (evaluating knowledge utility and consistency), (4) an oblivion procedure (discarding those patterns that seem useless) and (5) a consolidation process (promoting those patterns that are reliable and useful). The two first components are common in artificial intelligence (ML and automated deduction), but the latter three have usually been investigated in knowledge-based systems, cognitive science and informetrics (including bibliometrics and webometrics).

In this paper we develop a general framework that, given rule-based inductive and deductive subsystems, is able to constantly evaluate and arrange the rules in such a way that long-life ML becomes possible. The framework is based on a graph of deductive dependencies between examples and rules, from which several topological metrics are derived, such as support, certainty and significance. From these metrics we derive an oblivion mechanism to remove rules from working memory and a consolidation mechanism to promote rules to the long-term knowledge base. We evaluate the framework with a problem domain where several patterns about chess rules have to be learnt. We see how the system is able to keep the useful rules and perform differently when new tasks can take advantage of the knowledge that has been consolidated from previous tasks. To our knowledge, this is the first general and comprehensive framework for life-long ML that can be applied to a diversity of rule-based learning systems.

The rest of the paper is organised as follows. Section II presents the architecture of our framework and defines the metrics used to work with rules and knowledge. The experimental evaluation of our system is presented in Section III. Section IV reviews other related approaches in the literature. Finally, section V concludes the paper and outlines some future work.

\section{APPROACH}

The acquisition of learnt patterns after several tasks cannot be understood as a naive accumulation of what has been learnt. New rules can be redundant or inconsistent with old ones or may build upon previously acquired knowledge. Despite some notable efforts [1]-[3], the integration of ML with knowledge acquisition is still mostly unresolved. One of the key issues is that learning systems must be capable of learning new things (plasticity) without losing previously learned concepts (stability). This is called the The Stability-Plasticity dilemma [4].

In this work we take a different approach by considering that we start with an inductive engine (e.g., a rule learning algorithm, an inductive logic programming (ILP) system or an inductive programming (IP) system) and a deductive engine (e.g., a coverage checker, an automated deduction system or a declarative programming language) and, over them, we construct a long-life ML system (see Figure 1).

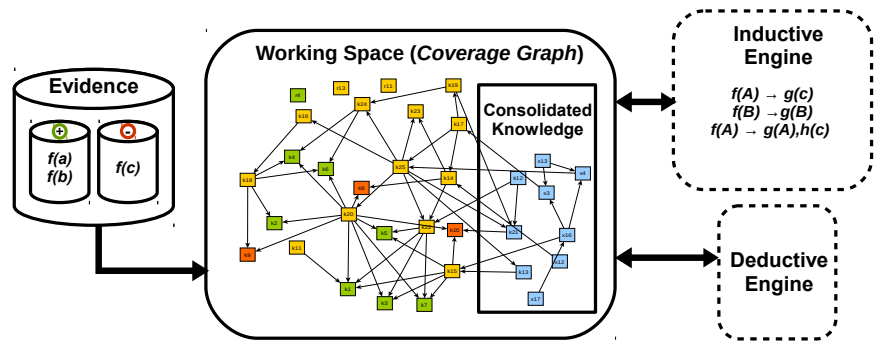

Fig. 1: Architecture of the long-life learning framework. 
For this purpose, several issues have to be addressed: (1) The inductive engine can generate many possible hypotheses and patterns. Once brought to working memory we require metrics to evaluate how these hypotheses behave and how they are related. (2) As working memory and computational time are limited, we need an oblivion criterion to discard some rules. (3) The deductive engine checks the coverage of each hypothesis independently, using the consolidated knowledge as auxiliary rules, but not other working rules. As a result, only when new knowledge is consolidated we can use it for new problems or for more difficult examples of the same problem. (4) The promotion of rules into consolidated knowledge must avoid unnecessarily large knowledge bases and the consolidation of rules that are useless, too preliminary or inconsistent. This means that rules must promoted and demoted.

One of our design considerations is that the approach should work with any inductive engine that is able to generate rules, such as association rule algorithms, IP systems such as $\mathrm{gErl}$ [5], or ILP systems such as Progol [6]. Actually, we must also provide a deductive engine for the representation language.

Let us now describe some notation that will be necessary to describe how the framework works in more detail.

\section{A. Notation}

We consider that 'rules' are used for expressing examples (denoted as $e$ or, alternatively, as $e^{+}$and $e^{-}$when we refer to positive and negative examples), patterns and background knowledge. The set of all possible rules is denoted by $\mathcal{R}$, where $R \subset \mathcal{R}$ is the working space or memory, and $B \subset \mathcal{R}$ is the background or consolidated knowledge base.

We represent the rules as vertexes or nodes $V$ in an acyclic directed graph $G(V, A)$ that we call coverage graph, because the directed edges $A$ represent the coverage relations between the different rules ${ }^{1}$ as determined by the deductive engine. Hence, if there is an edge $a=(x, y)$ (or $x \rightarrow y$ ), then $y$ is said to be directly covered by $x$ using $B^{2}$. The set of ancestors and successors of a node $v$ are defined as $\operatorname{anc}(v)=\{x \mid x \rightarrow v\}$ and $\operatorname{suc}(v)=\{y \mid v \rightarrow y\}$ (respectively). Figure 2 shows an example of Coverage Graph of a well-known ILP problem: family relationship. In this problem, the task is to define the target relation daughter $(X, Y)$, which states that person $X$ is daughter of person $Y$. The problem consists of three positive examples, two negative ones, and seven selected rules that try to generalise and solve the problem (Table I right), whereas $B$ is composed of the relations female and parent (Table I left). Note that the rules in $B$ have not been included in the graph for clarity, although they belong to the initial consolidated knowledge.

\section{B. Metrics}

From the coverage graph, we derive several indicators to determine which rules are more relevant, useful and consistent.

\footnotetext{
${ }^{1}$ We say that a rule $\rho_{a}$ is covered by another rule $\rho_{b}$ if $\rho_{b} \theta$-subsumes $\rho_{a}$, namely, $\rho_{b} \prec \rho_{a}$ iff exists an substitution $\theta$ such that $\rho_{b} \theta \subseteq \rho_{a}$.

${ }^{2}$ For simplicity, the coverage graphs do not include the edges for the transitive closure of the covering relation, i.e., if a node $x$ covers nodes $y$ and $z$, but $y$ also covers $z$, only the edges $x \rightarrow y$ and $y \rightarrow z$ are included.
}

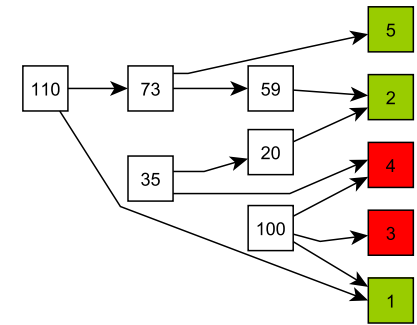

Fig. 2: Coverage Graph of the family relations problem. Green and red nodes refer to positive and negative examples respectively. The graph shows rule IDs according to Table I.

\begin{tabular}{|c|c|c|c|}
\hline \multicolumn{2}{|c|}{ Background Knowledge } & \multicolumn{2}{|r|}{ Rules } \\
\hline ID & Rule & ID & Rule \\
\hline k1 & parent(ann, mary) & 1 & daughter(mary,ann) \\
\hline $\mathrm{k} 2$ & parent(ann, tom) & 2 & daughter(eve,tom) \\
\hline $\mathrm{k} 3$ & parent(tom, eve) & 3 & daughter(tom,ann) \\
\hline $\mathrm{k} 4$ & parent(tom, ian) & 4 & daughter(eve,ann) \\
\hline $\mathrm{k} 5$ & female(ann) & 5 & daughter(cris,tom) \\
\hline k6 & female(mary) & 100 & daughter $(\mathrm{X}, \mathrm{Y}) \leftarrow$ female $(\mathrm{Y})$, parent(Y,mary) \\
\hline \multirow[t]{5}{*}{ k7 } & female(eve) & 59 & daughter(eve,tom) $\leftarrow$ female (eve),parent(tom,eve) \\
\hline & & 20 & daughter(eve,tom $) \leftarrow$ female (eve) \\
\hline & & 35 & daughter $($ eve, $\mathrm{Y}) \leftarrow$ female $($ eve $)$ \\
\hline & & & daughter $(\mathrm{X}$, tom $) \leftarrow$ female $(\mathrm{X})$,parent $($ tom, $\mathrm{X})$ \\
\hline & & 110 & daughter(X,Y) $\leftarrow$ female $(\mathrm{X})$,parent $(\mathrm{Y}, \mathrm{X})$ \\
\hline
\end{tabular}

TABLE I: Left: Background Knowledge for the family relations problem. Right: Rules of this problem in Prolog notation.

To illustrate these metrics, Table II shows some measures of significance and interestingness for the graph in Figure 2.

\begin{tabular}{rrrrrrrrr}
\hline ID & Size & Cert+ & Cert- & Sup+ & Sup- & Perm & Cons & Opt \\
\hline 1 & 18.429 & 1 & 0 & 1 & 0 & 0.755 & true & 1 \\
\hline 2 & 18.429 & 1 & 0 & 1 & 0 & 0.823 & true & 1 \\
\hline 3 & 18.429 & 0 & -1 & 0 & -1 & 0.755 & false & -1 \\
\hline 4 & 18.429 & 0 & -1 & 0 & -1 & 0.755 & false & -1 \\
\hline 5 & 18.429 & 1 & 0 & 1 & 0 & 0.12 & true & 1 \\
\hline 100 & 7.562 & 0.462 & -0.761 & 0.5 & -1.5 & 0.431 & false & -0.299 \\
\hline 59 & 18.791 & 0.462 & 0 & 0.5 & 0 & -0.649 & false & 0.231 \\
\hline 20 & 11.591 & 0.462 & 0 & 0.5 & 0 & 0.317 & false & 0.231 \\
\hline 35 & 8.784 & 0.226 & -0.462 & 0.5 & -0.5 & 0.113 & false & 0 \\
\hline 73 & 10.369 & 0.623 & 0 & 1.5 & 0 & 0.042 & true & 0.934 \\
\hline 110 & 4.754 & 0.494 & 0 & 2 & 0 & 1.198 & true & 0.988 \\
\hline
\end{tabular}

TABLE II: Metrics for the rules on the right side of Table I.

1) Support: The support of a rule aims at representing how important a rule is in terms of coverage: the higher number of examples covered, the higher the support value is. All the rules have two kinds of support, positive and negative. The examples have a fixed support value $\operatorname{Sup}_{f}$ that is given initially as: $\operatorname{Sup}_{f}^{+}\left(e^{+}\right)=1, \operatorname{Sup}_{f}^{-}\left(e^{+}\right)=0, \operatorname{Sup}_{f}^{+}\left(e^{-}\right)=0$ and $\operatorname{Sup}_{f}^{-}\left(e^{-}\right)=-1$. For the rest of nodes, $\operatorname{Sup}_{f}^{+}(v)$ and $\operatorname{Sup}_{f}^{-}(v)$ are 0 . From here, we propagate the support through the acyclic graph backwards:

$$
\begin{aligned}
& \operatorname{Sup}^{+}(\rho)=\operatorname{Sup}_{f}^{+}(\rho)+\sum_{\nu \in \operatorname{suc}(\rho)} \frac{\operatorname{Sup}^{+}(\nu)}{|\operatorname{Suc}(\nu)|} \\
& \operatorname{Sup}^{-}(\rho)=\operatorname{Sup}_{f}^{-}(\rho)+\sum_{\nu \in \operatorname{suc}(\rho)} \frac{\operatorname{Sunc}(\nu) \mid}{\mid \operatorname{anc}}
\end{aligned}
$$

Note that as we divide the support of the outcoming nodes by $|\operatorname{anc}(\nu)|$, the support is conservative. This means that the positive and negative supports of rule $\rho$ are always an underestimate of how many positive and negative rules, respectively, $\rho$ covers. For instance, the positive example with $\mathrm{ID}=1$ in Figure 2 is covered by the rules with IDs 100 and 110, so both of them receive half of its support (a positive support equal to 
0.5 and a negative support equal to 0 ). The reason for this is that if too many rules are covering the same examples, their support is shared and reduced, thus avoiding many rules for a few examples.

2) Certainty or groundedness: The certainty or groundedness of a rule means how much we can trust in it, and its value decreases as the length of the paths that connect the rule to the example increases. This is natural since a concept that is too far from the actual examples is more general than them and, thus, it is less grounded. All the rules have two kinds of groundedness, the positive and the negative one. The examples have, in general, the highest possible certainty (they are the only ones in which we can trust completely, assuming a learning scenario without noise). $\mathrm{Cert}_{f}$ is a fixed value assigned to the examples with the initial values $\operatorname{Cert}_{f}^{+}\left(e^{+}\right)=1, \operatorname{Cert}_{f}^{-}\left(e^{+}\right)=0, \operatorname{Cert}_{f}^{+}\left(e^{-}\right)=0$ and $\operatorname{Cert}_{f}^{-}\left(e^{-}\right)=-1$. As we want positive certainty to go between 0 and 1 and negative certainty between -1 and 0 , we use a logistic function $L(x)=\frac{1}{1+e^{-x}}$ :

$$
\begin{aligned}
& \operatorname{Cert}^{+}(\rho)=2 L\left(\operatorname{Cert}_{f}^{+}(\rho)+\sum_{\nu \in \operatorname{suc}(\rho)} \operatorname{Cert}^{+}(\nu)\right)-1 \\
& \operatorname{Cert}^{-}(\rho)=2 L\left(\operatorname{Cert}_{f}^{-}(\rho)+\sum_{\nu \in \operatorname{suc}(\rho)} \operatorname{Cert}^{-}(\nu)\right)-1
\end{aligned}
$$

3) Optimality or Significance: Support and certainty are related metrics but are focussed on different things, in a similar way as support and confidence in association rules. With the aim of integrating support and certainty, we define the optimality or significance of a rule $\rho$ :

$$
\operatorname{Opt}(\rho)= \begin{cases}-(\operatorname{Sup}(\rho)) \cdot \operatorname{Cert}(\rho)) & \text { if }(\operatorname{Sup}(\rho)<0) \wedge(\operatorname{Cert}(\rho)<0) \\ \operatorname{Sup}(\rho) \cdot \operatorname{Cert}(\rho) & \text { otherwise }\end{cases}
$$

where $\operatorname{Sup}(\rho)=\operatorname{Sup}^{+}(\rho)+\operatorname{Sup}^{-}(\rho)$ and $\operatorname{Cert}(\rho)=$ $\operatorname{Cert}^{+}(\rho)+\operatorname{Cert}^{-}(\rho)$.

This formula tries to emphasise differences between the positive and negative variants of Sup and Cert.

\section{Working space: oblivion mechanism}

The optimality of a rule $\rho$ is a core metric to determine its usefulness, but it is also important to see if $\rho$ is covered by another rule of higher optimality. If it is the case, $\rho$ is mostly redundant and it could be discarded safely. This idea leads to the following definition for the permanence of a rule:

$$
\operatorname{Perm}(\rho)=\operatorname{Opt}(\rho)-\underset{\nu \in \operatorname{anc}(\rho)}{\arg \max } \operatorname{Opt}(\nu)+\frac{1}{\operatorname{size}(\rho)}
$$

where size refers to the size (in bits) of the rule (definition available at [7]). Inspired by the MML philosophy [8], [9], the last term allows us to give more preference to those rules that are shorter.

The lower the value of permanence a rule has, the higher possibilities it has to be forgotten, depending on an oblivion threshold. Figure 3 shows the evolution of the coverage graph in Figure 2 through four oblivion steps ${ }^{3}$. Due to space limitation, we do not include in the paper the measures for the rules,

\footnotetext{
${ }^{3}$ In this example, we have forgotten one rule at a time, but the actual pace and number of rules to forget can be tuned to the purpose of the system. For instance, oblivion can be triggered when the number of rules exceeds a given threshold and several rules can be forgotten at a time. In this way, the graph and the metrics do not have to be recalculated for each step of the system.
}

which are available at [7]. Following with the example, in step 1 we see that rule number 59 is redundant because it is covered by a more significant rule (with ID $110, \operatorname{Opt}(110)=1.34$, $O p t(59)=0.231)$, and it has the lowest value of permanence $(\operatorname{Perm}(59)=-0.649$ and $\operatorname{Perm}(110)=1.55)$. Thus, rule 59 is forgotten, the coverage graph is redrawn and the metrics are recalculated.

In case there is an oblivion step that deletes any leaf node, its support is distributed equally among the rules that cover it, and added to the "fixed" or intrinsic support of these rules. Similarly, the certainty and its fixed values are also 'inherited' upwards (but not distributed).

\section{Consolidated knowledge: promotion and demotion}

Some of the rules with good indicators in the working space have to be eventually promoted to consolidated knowledge (or belief). This has to be a careful process, as the consolidated knowledge will be used by the deductive engine to calculate coverage. This means that an inconsistent rule that is promoted to the consolidated knowledge may have important consequences on the behaviour of the system.

We will set a threshold $\theta_{p}$ on the optimality to consolidate or promote a rule to a belief status. $B$ is therefore continuously grown with new rules. The promotion system is mirrored by a demotion system, with the use of another threshold $\theta_{d}$ such that if any rule in $B$ has a lower value of optimality, then the rule is demoted to the working space. The original background knowledge $\left(B_{0}\right)$ cannot be demoted (and forgotten).

In the example in Figure 3, we have established $\theta_{p}$ equal to the average optimality (using the absolute value $|\operatorname{Opt}(\rho)|$ before averaging) of all the rules in the working space. Then, in step 1 , all the rules that exceed this average value $(0.695)$ will be consolidated to the background base (rules 1, 2, 5, 73 and 110). Any rule that is promoted to the consolidation step cannot be target of the oblivion mechanism until it is demoted to the working space again (in the example, we have considered a demoting threshold $\theta_{d}$ equal to $\theta_{p}$ ). Thus, in step 2 , rule 1 has the lowest permanence value $(\operatorname{Perm}(1)=$ $-0.357)$ but $35(\operatorname{Perm}(35)=-0.113)$ is forgotten instead, because the former is a consolidated rule.

\section{EXPERIMENTS}

As mentioned in section II, one of the issues in other learning systems is the Stability-Plasticity dilemma. We claim that our approach is able to address this issue in a lifelong learning process. For this purpose, we have conducted an experimental evaluation to explore the following questions: (a) is it possible to generate a large repository of consolidated knowledge assessing the usefulness of the rules? and (b) is our approach able to forget or revise the existing knowledge in order to generate a reusable knowledge base? We illustrate these features in one single domain. The ultimate goal of these experiments is to see whether the framework is general enough to work with any inductive and deductive engine. Additional information not included in the paper about the rules and their measures for each experiment is available at [7]. 


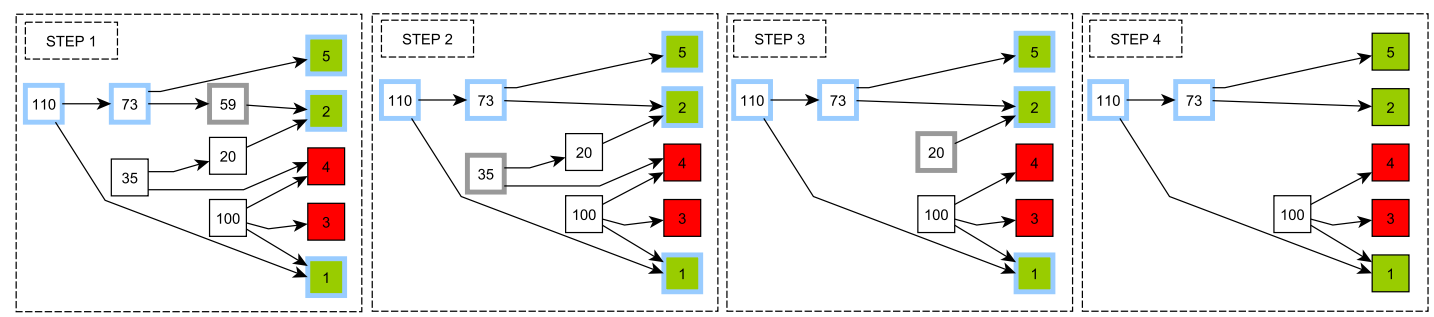

Fig. 3: Coverage Graph of the family problem. Green and red nodes refer to positive and negative examples respectively. Nodes with a thick grey square represent the rules to be forgotten. Nodes with a thick blue square represent consolidated rules.

\section{A. Methodology}

We focus on learning a model of legal moves of different pieces of chess from a set of legal (positive) and illegal (negative) move examples (extracted from [10]). Each example corresponds to a move on an empty board of a specific piece, and is represented by a triple from the domain Piece $\times$ Pos $\times$ Pos, where the second and third components are, respectively, the piece's initial position and its destination on the chessboard. Positions are expressed as tuples from the domain File $\times$ Rank where files (a-h) stand for columns and ranks (1-8) stand for rows. For instance, using a Prolog notation (as in the example in the previous section), the fact move (knight, pos $(d, 5)$, pos $(e, 3))$ represents a legal move of the knight from position $(\mathrm{d}, 5)$ to $(\mathrm{e}, 3)$. The only background predicate used is the absolute difference, $\operatorname{diff}(X, Y)$, that calculates the distance between $X$ and $Y$, where both $X$ and $Y$ can be ranks or files (see Table III).

\begin{tabular}{|c|c|c|c|}
\hline ID & Rule & ID & Rule \\
\hline & project(a,1). & \multirow{4}{*}{ K11 } & rdiff(Rank1,Rank2,Diff) :- \\
\hline K2 & project(b,2). & & $\operatorname{rank}(\operatorname{Rank} 1), \operatorname{rank}(\operatorname{Rank} 2)$ \\
\hline K3 & project(c,3). & & Diff1 is Rank1-Rank2, \\
\hline K4 & $\operatorname{project}(\mathrm{d}, 4)$. & & abs(Diff1,Diff). \\
\hline K5 & project(e,5). & \multirow{6}{*}{ K12 } & \multirow{6}{*}{$\begin{array}{l}\text { fdiff(File1,File2,Diff) :- } \\
\text { file(File1), file(File2), } \\
\text { project(File1,Rank1), } \\
\text { project(File2,Rank2), } \\
\text { Diff1 is Rank1-Rank2, } \\
\text { abs(Diff1,Diff). }\end{array}$} \\
\hline K6 & project(f,6). & & \\
\hline K7 & project $(\mathrm{g}, 7)$. & & \\
\hline K8 & project(h,8). & & \\
\hline K9 & $\operatorname{abs}(X, X):-X>=0$ & & \\
\hline & $\operatorname{abs}(\mathrm{X}, \mathrm{Y}):-\mathrm{X}<0, \mathrm{Y}$ is $-\mathrm{X}$. & & \\
\hline
\end{tabular}

TABLE III: Background knowledge for the chess problem.

A random set of 50 chess moves (positive and negative examples) from all chess pieces except the pawn (rook, bishop, knight, queen and king) is given. We also consider that an inductive engine is generating rules during the whole process and they are arriving to the system in a random order. In our case, we have taken the rules generated by the ILP system Progol [6] (50 in total). To determine how many examples and rules are given to the system for each step, we use (with replacement $\left.{ }^{4}\right)$ a geometric distribution $\operatorname{Pr}(X=k)=(1-$ $p)^{k-1} \cdot p$, where $k$ is the number of examples or rules, and $p$ is the probability of success (we set it to 0.5 ).

In the first experiment, we show how the oblivion mechanism works. This tries to represent a situation where we have a limited working space, for which we have considered that the maximum number of rules in it is 60 . Hence, every time this value is exceeded the oblivion process is launched, forgetting

\footnotetext{
${ }^{4}$ This allows us to better mimic a situation where a (usually memory-less) inductive engine can produce rules it has already generated.
}

up to 50\% of the most meaningless rules (lowest Perm value). Also, we have set the consolidation criterion for rules to those above a threshold of significance (optimality) greater than 1.0 (the average of the absolute value of the example optimalities).

Figure 4 shows the evolution of the system in 500 steps. Now, the variations in the amount of consolidated rules (dotted grey line) and rules in the working space (dotted yellow line) allows us to observe how the oblivion mechanism works (every 30 steps approximately). At step 500, the system reaches a stable situation in which the number of consolidated rules and the average optimality of all the rules in the working space becomes almost constant. The appearance of new rules in the system or the execution of the oblivion mechanism has a slight effect, with the exception of the average optimality of the consolidated rules that is highly correlated with the oblivion mechanism: every time it runs, the working space is cleaned of useless rules. This strongly affects the metrics of the consolidated rules (and to a lesser extent to the whole set of rules), which have to be recalculated.

The second experiment we carried out tries to show the capability of our approach to learn of new knowledge from previously consolidated concepts. This experiment is divided into two phases. In the first phase we provided the system with the rules and examples of moves of the rook and bishop (15 and 30 rules respectively). The consolidation criterion has not been changed, but the maximum number of rules in the working space has been reduced to 15 and also the percentage of meaningless rules that are forgotten for each oblivion process $(30 \%)$. In the first 100 steps of Figure 5 we can see that, due to the lower maximum number of rules allowed in the working space and the lower percentage of rules forgotten, the oblivion mechanism runs here every few steps, showing non-constant sawtooth-like wave ramps for the number of rules in the working system (dotted yellow). However, the number of consolidated rules remains constant almost from the beginning. The set of consolidated rules after 100 steps perfectly generalises all the legal moves of the rook and the bishop pieces (see Table IV). In the second phase, we provided the system with a new set of rules and examples (10 and 20 rules respectively) only representing moves of the queen. Apart from using the background knowledge that is provided initially, it should also be possible to use the previously learnt general moves from the rook and the bishop in order to express the moves of the queen. This is what the inductive engine can take advantage of. Table $\mathrm{V}$ shows the set of consolidated rules which consists of the previously learnt 


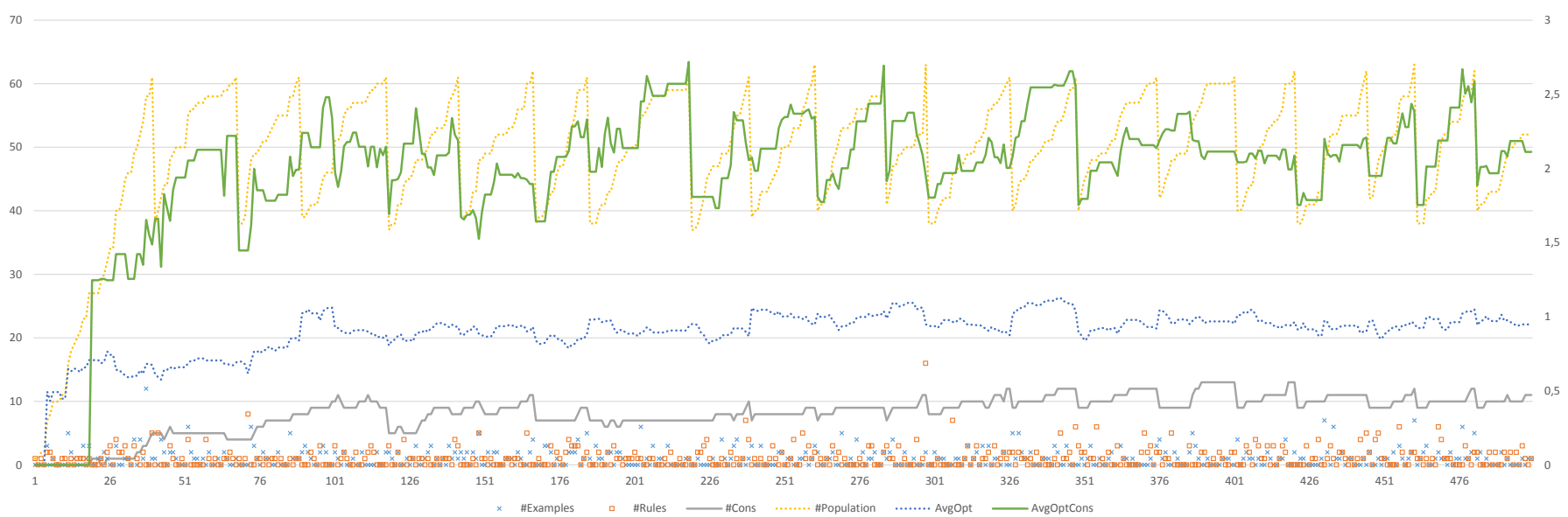

Fig. 4: Evolution of the chess problem: \#Examples and \#Rules show the examples that arrive and the rules that are generated by the inductive engine for each step, \#Cons shows how many rules there are in the consolidate knowledge (initially the background knowledge), \#Population shows the total number of rules, AvgOpt shows the average optimality for all rules and AvgOptCons the average optimality for all consolidated rules.

rules that generalise the legal moves from the rook and bishop, and a new set of rules that represents the legal moves of the queen. This latter set includes a pair of rules ( $q 29$ and $q 25)$ that use the rook and bishop rules and represent all the possible moves of the queen: $q 25$, which covers both the horizontal and vertical moves of the queen; and $q 29$, which covers the same move as the rule $q 23$ (diagonal movement) but it is more likely to be forgotten in case of demotion due to its larger size.

\section{RELATED WORK}

The results of the experiments presented in Section III show that our approach is able of incremental and continual learning based on the reuse of knowledge. This is the context of Lifelong Machine Learning [11], or LML, and related to areas such as Multiple Task Learning, Transfer Learning, Reinforcement Learning or Incremental Learning, have similar objectives.

One distinctive trait of our approach is that we use a rule-based approach and knowledge is arranged by coverage. This is different to other lifelong learning approaches that use explanation-based neural networks (EBNN) [12], backpropagation neural networks [13], [14] and reinforcement learning approaches [15]. Another important difference is that here we do not work with a task-to-task mapping as in transfer learning.

Closer to our approach we find ELLA (Efficient Lifelong Learning Algorithm) [16] and NELL (Never-Ending Language Learner) [17], which are another approaches to LML able to consolidate knowledge within a long-term domain knowledge structure. However they do not introduce oblivion mechanisms that could eliminate obsolete knowledge and lack the layered view of knowledge of a covering graph. Henderson [18] presents an inductive inference system that automatically acquires knowledge employed for solving harder problems through experience of solving easier tasks. This work is centred on abstract learning and the generalisation of rules, but does not grow the existing knowledge base systematically.

From a very different point of view, our coverage graph is also highly influenced by those problems related to link analysis in web graphs such as the HITS algorithm [19], PageRank [20] or SALSA [21]. Other related ideas appear in areas of informetrics, but in our case it is not reputation but explanatory relevance and consistency what matter.

\section{CONCLUSIONS}

In this paper we have presented a new framework to deal with lifelong machine learning, where knowledge is promoted from working space to consolidated knowledge (and vice versa). We have also introduced an oblivion mechanism to discard those rules that are useless. The framework is modular and easily adaptable to many inductive engines that are able to output rules (or other units of knowledge) provided that we have a deductive engine to check coverage between the rules, in order to create the coverage graph. The use of oblivion criteria can be used to help tuning the system according to how much space there is available, and most especially, about how many rules can be processed in a reasonable time by the deductive engine, as the working space graph needs to be recalculated for every step.

The goal of this work was to evaluate the framework in general terms. From the experiments we have seen that the metrics and thresholds work reasonably well. Additional evaluation is needed with more and different problems and other inductive and deductive engines in order to get further information about the quality of the metrics we have defined and the use of thresholds for forgetting, promoting and demoting.

\section{REFERENCES}

[1] E. Sommer, K. Morik, J.-M. Andr, and M. Uszynski, "What online machine learning can do for knowledge acquisitiona case study," Knowledge Acquisition, vol. 6, no. 4, pp. 435 - 460, 1994.

[2] E. Sommer, "Fender: An approach to theory restructuring," in Machine Learning: ECML-95. Springer, 1995, pp. 356-359.

[3] G. I. Webb, J. Wells, and Z. Zheng, "An experimental evaluation of integrating machine learning with knowledge acquisition," Machine Learning, vol. 35, no. 1, pp. 5-23, 1999. 


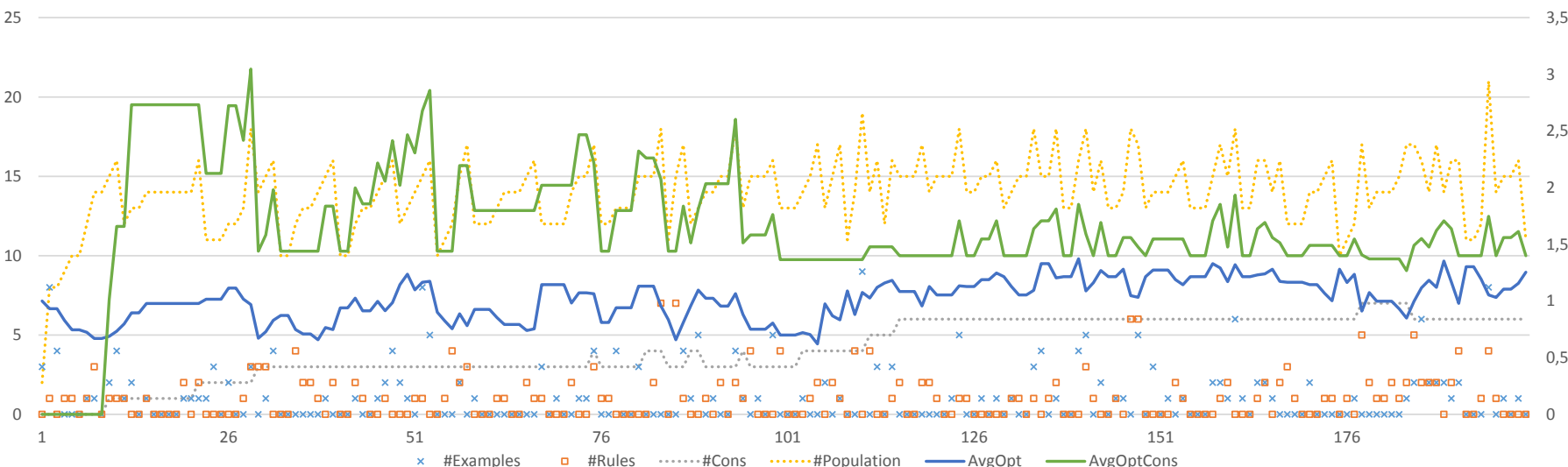

Fig. 5: Evolution of the same indicators as in Figure 4 for the incremental chess problem (rook and bishop moves in the first 100 steps, and queen moves in the following 100 steps). We see a non-constant sawtooth-like picture for the number of rules in the working space whereas the consolidated rules became constant in each different learning process, and the global optimality becomes stable between 1 and 1.5.

\begin{tabular}{llrrrrrrrr}
\hline ID & \multicolumn{1}{c}{ Rule } & Size & Cert $^{+}$ & Cert $^{-}$ & Sup $^{+}$ & Sup & Perm & Cons & Opt $^{-}$ \\
\hline b10 & move(bishop,pos(A,B),pos(C,D)) :- rdiff(B,D,E), fdiff(A,C,E). & 7.924 & 0.905 & 0.0 & 1.833 & 0.0 & 1.784 & true & 1.658 \\
\hline r15 & move(rook,pos(A,B),pos(A,C)). & 18.133 & 0.761 & 0.0 & 2.0 & 0.0 & 1.419 & true & 1.522 \\
\hline r16 & move(rook,pos(A,B),pos(C,B)). & 18.133 & 0.761 & 0.0 & 1.5 & 0.0 & 1.038 & true & 1.141
\end{tabular}

TABLE IV: Consolidated rules and metrics (from left to right: size, positive and negative certainty, positive and negative support, permanence, is consolidated?, and optimality) for the chess problem (rook and bishop moves) at step 100. All the legal moves for these pieces are covered by the rules and no better rules can be obtained.

\begin{tabular}{|c|c|c|c|c|c|c|c|c|c|}
\hline ID & Rule & Size & Cert $^{+}$ & Cert $^{-}$ & Sup $^{+}$ & Sup $^{-}$ & Perm & Cons & Opt \\
\hline$b 10$ & move(bishop,pos(A,B),pos(C,D)) & 7.924 & 0.905 & 0.0 & 1.833 & 0.0 & 1.784 & true & 1.658 \\
\hline $\mathrm{r} 15$ & move(rook, pos(A,B),pos(A,C)). & 18.133 & 0.761 & 0.0 & 2.0 & 0.0 & 1.577 & true & 1.522 \\
\hline r16 & move(rook,pos(A,B),pos(C,B)). & 18.133 & 0.761 & 0.0 & 1.5 & 0.0 & 1.196 & true & 1.141 \\
\hline 23 & move(queen,pos(A,B),pos(C,D)) & 12.384 & 0.905 & 0.0 & 1.333 & 0.0 & 1.286 & le & 1.206 \\
\hline$q 29$ & move(queen,pos(A,B),pos(C,D)) :- move(bishop,pos( & 18.428 & 0.905 & 0.0 & 1.333 & 0.0 & 1.26 & true & 1.206 \\
\hline 25 & move(queen,pos(A,B),pos(C,D)) :- move(rook,pos(A,B),pos(C,D)) & 18.428 & 0.964 & 0.0 & 2.0 & 0.0 & 1.982 & true & 1.928 \\
\hline
\end{tabular}

TABLE V: Consolidated rules and metrics (same as in Table IV) for the chess problem at step 200 (the 100 firsts steps for learning the rook and bishop moves, and the 100 following steps for learning the queen moves). All queen legal moves are covered by taking advantage of previously learned moves of rook and bishop pieces, whose legal moves are also covered.

[4] M. Mermillod, A. Bugaiska, and P. Bonin, "The stability-plasticity dilemma: investigating the continuum from catastrophic forgetting to age-limited learning effects," Frontiers in psychology, vol. 4, 2013.

[5] F. Martínez-Plumed, C. Ferri, J. Hernández-Orallo, and M. RamírezQuintana, "Learning with configurable operators and RL-based heuristics," in New Frontiers in Mining Complex Patterns, ser. Lecture Notes in Computer Science, 2013, vol. 7765, pp. 1-16.

[6] S. Muggleton, "Inverse entailment and progol," New Generation Computing, vol. 13 , no. 3-4, pp. 245-286, 1995.

[7] F. Martínez-Plumed, C. Ferri, J. Hernández-Orallo, and M. RamírezQuintana, "Coverage graph metrics, consolidation and oblivion mechanisms for lifelong machine learning," Tech. Rep., 2014. [Online]. Available: http://users.dsic.upv.es/ fmartinez/papers/ICMLA14_TR.pdf

[8] M. Li and P. M. Vitányi, An Introduction to Kolmogorov Complexity and Its Applications, 3rd ed. Springer Publishing Company, 2008.

[9] C. Wallace, Statistical and Inductive Inference by Minimum Message Length (Information Science and Statistics). Springer, 2005.

[10] S. Muggleton, M. Bain, J. Hayes-michie, and D. Michie, "An experimental comparison of human and machine learning formalisms," in In Proc. of 6th International Workshop on Machine Learning. Morgan Kaufmann, 1989, pp. 113-118.

[11] S. Thrun, "Is learning the n-th thing any easier than learning the first," in Advances in Neural Information Processing Systems, vol. 8, 1996, pp. 640-646.

[12] — Explanation-Based Neural Network Learning: A Lifelong Learning Approach. Boston, MA: Kluwer Academic Publishers, 1996.

[13] D. L. Silver and R. E. Mercer, "The parallel transfer of task knowledge using dynamic learning rates based on a measure of relatedness," in Connection Science Special Issue: Transfer in Inductive Systems, 1996, pp. 277-294.

[14] D. L. Silver and R. Poirier, "Machine life-long learning with csmtl networks." in AAAI. AAAI Press, 2006.

[15] M. B. Ring, "Child: A first step towards continual learning," in Machine Learning, 1997, pp. 77-104.

[16] E. Eaton and P. L. Ruvolo, "Ella: An efficient lifelong learning algorithm," in Proceedings of the 30th International Conference on Machine Learning (ICML-13), vol. 28, 2013, pp. 507-515.

[17] A. Carlson, J. Betteridge, B. Kisiel, B. Settles, E. R. H. Jr., and T. M. Mitchell, "Toward an architecture for never-ending language learning," in Proceedings of the Twenty-Fourth Conference on Artificial Intelligence (AAAI 2010), 2010.

[18] R. Henderson, "Cumulative learning in the lambda calculus," Ph.D. dissertation, Imperial College London, 2014.

[19] J. M. Kleinberg, "Authoritative sources in a hyperlinked environment," J. ACM, vol. 46, no. 5, pp. 604-632, Sep. 1999.

[20] S. Brin and L. Page, "The anatomy of a large-scale hypertextual web search engine," Comput. Netw. ISDN Syst., vol. 30, no. 1-7, pp. 107-117, Apr. 1998

[21] R. Lempel and S. Moran, "The stochastic approach for link-structure analysis (salsa) and the tkc effect," Comput. Netw., vol. 33, no. 1-6, pp. 387-401, Jun. 2000. 